\title{
Near infrared spectroscopy: a new tool for studying physical and chemical properties of polysaccharide gels
}

\author{
Yiqun Huang ${ }^{\mathrm{a}}$, Juming Tang ${ }^{\mathrm{a}, *}$, Barry G. Swanson ${ }^{\mathrm{b}}$, Anna G. Cavinato ${ }^{\mathrm{c}}$, \\ Mengshi Lin ${ }^{\mathrm{b}}$, Barbara A. Rasco ${ }^{\mathrm{b}}$ \\ ${ }^{a}$ Department of Biological System Engineering, Washington State University, 213 L.J. Smith Hall, P.O. Box 646120, Pullman, WA 99164-6120, USA \\ ${ }^{\mathrm{b}}$ Department of Food Science and Human Nutrition, Washington State University, P.O. Box 646376, Pullman, WA 99164-6376, USA \\ ${ }^{\mathrm{c}}$ Chemistry Program, Eastern Oregon University, One University Boulevard, La Grande, OR 97850, USA
}

Received 24 November 2002; revised 4 February 2003; accepted 7 February 2003

\begin{abstract}
This study explored the use of short-wavelength near infrared spectroscopy (SW-NIR, 600-1100 nm) to characterize gellan gel, a polysaccharide gel. SW-NIR can classify commercial low acyl (LA) and high acyl (HA) gellan gel mixtures and predict the ratio of LA and HA gellan in a gel mixture $\left(R^{2}=0.93\right.$, standard error of prediction $\left.=9.19 \%\right)$. In addition, SW-NIR methods can classify LA gellan gels based upon storage temperature $\left(4\right.$ and $\left.22^{\circ} \mathrm{C}\right)$ and can determine gelling temperature of LA gellan gel. SW-NIR methods demonstrated that the structure of LA gellan gel $\left(4 \mathrm{mM} \mathrm{Ca}^{++}\right)$remained stable under room temperature following gelation $(5-72 \mathrm{~h})$.

(C) 2003 Elsevier Science Ltd. All rights reserved.
\end{abstract}

Keywords: Near infrared; Gellan; Gelling temperature; Storage temperature; Storage time; Gel classification

\section{Introduction}

Interest in using near infrared (NIR, 700-2500 nm) as an analytical tool can be traced back to the 1940s. Because of the potential for rapid and non-destructive identification and quantification of analytes in complex matrices, NIR methods were widely used for agricultural and pharmaceutical applications over the past three decades.

Unlike mid-infrared $(2500 \mathrm{~nm}-25 \mu \mathrm{m})$ spectra, NIR spectra are composed primarily of overtone and combination bands. Absorption in the NIR region is mainly due to the hydrogen stretching vibrations, involving $\mathrm{C}-\mathrm{H}, \mathrm{O}-\mathrm{H}$ and $\mathrm{N}-\mathrm{H}$ containing functional groups. For pure water, a prominent water band at around $970 \mathrm{~nm}$ is observed in a typical short-wavelength near infrared spectrum (SW-NIR: 700-1100 nm) (Phelan, Barlow, Kelly, Jinguji, \& Callis, 1989). Several other water bands around 1450, 1950 and $2250 \mathrm{~nm}$ are observed in a long-wavelength NIR (1100$2500 \mathrm{~nm}$ ) spectrum (Lin \& Brown, 1992). Food hydrocolloids contain large amount of water and NIR spectra of

\footnotetext{
* Corresponding author. Tel.: + 1-509-335-2140; fax: + 1-509-335-2722.

E-mail address: jtang@wsu.edu (J. Tang).
}

hydrocolloids generally exhibit prominent water features (Huang, Cavinato, Mayes, Bledsoe, \& Rasco, 2002).

A major advantage of SW-NIR compared to other spectral regions, such as mid-infrared, is that it allows for the use of longer path lengths, which simplifies sampling and handling procedures and makes non-invasive measurements in intact food systems possible. In addition, the use of fiber optic probes for NIR analysis makes it possible to remotely collect sample spectra.

NIR methods are successfully applied in polymer science, including detection of single molecules in flowing sample streams (Soper \& Legendre, 1998), detection of monomer droplets in polymer latex (Vieira, Sayer, Lima, \& Pinto, 2001), characterization of polymer melt during extrusion processing (Rohe, Kolle, Stern, Eisenreich, \& Eyerer, 2001), determination of polymer compositions and kinetics of polymerization of ethylene (Camacho \& Karlsson, 2001, Nees \& Buback, 1976). However, to the best of our knowledge, there are no reports using NIR to study polymer gelling temperature or studying the physical or chemical properties of hydrocolloids used in foods.

Because variation in rheological properties, such as gelling temperature and yield stress, are ultimately controlled by chemical interactions of water and 
hydrocolloid, it should be possible to use NIR to monitor changes in these properties in a food hydrocolloid such as gellan gel.

Gellan is a linear anionic polysaccharide gelling agent consisting of tetrasaccharide repeat units. Gellan was recently approved as a food hydrocolloid by the FDA and is commercially available in two forms: low acyl (LA) and a high acyl (HA) gellan powder. HA gellan gel differs from LA gellan gel by having a substitution of a glycerate group and an acetate group (50\%) at $\mathrm{O}_{2}$ and $\mathrm{O}_{6}$ of one glucose residue in each tetrasaccharide unit. HA gellan forms soft and elastic gels, while LA gellan forms strong and brittle gels. The mixture of these two gellan forms can provide gels with a wide range of intermediate properties depending upon the ratio of LA/HA gellan.

It is a common practice to add ions or other compounds such as sugar or citric acid to a hydrocolloid system to modify gel forming and sensory properties. Although ions or other compounds may have no absorption bands in the NIR region, their presence induces changes in the hydrogen bonding within water molecules (Lin \& Brown, 1992) and alters the shape and position of water bands in the NIR region. Therefore, these ions induced modifications of water-water interactions and water-colloid interaction should be apparent as changes in NIR spectral features.

Temperature variations also strongly affect the hydrogen bonding properties of water (Finch \& Lippincott, 1956; Lin \& Brown, 1992) and alter the interactions between water molecules and hydrocolloids. Such changes caused by temperature are apparent in NIR spectra (Huang, 2001; Lin \& Brown, 1992).

The objectives of this study were to explore the use of NIR spectroscopy to characterize commercial LA and HA gellan gels and mixtures of these two gels, including the use of SW-NIR to differentiate LA and HA gellan gels and gel mixtures, to detect the effects of storage time and storage temperature on the changes of physical and chemical properties of gellan gels, and to determine the gelling temperature.

\section{Materials and methods}

\subsection{Sample preparation and spectral measurement}

Two commercial gellan powders, LA (Kelcogel F) and HA (Kelcogel LT100) gellan powder were provided by CP Kelco US, Inc. (Wilmington, DE). One gram of gellan powder was dispersed into $100 \mathrm{ml}$ of deionized water in a pre-weighed $250 \mathrm{ml}$ beaker and heated to boiling within 15 min with constant magnetic stirring. The gellan dispersion was then weighed and hot water added to compensate for the water lost during heating. Simultaneously, calcium chloride dehydrate crystals (ACS reagent, Mallinckrodt Baker, Inc., Phillipsburg, NJ) was added to prepare gellan dispersions with calcium concentrations ranging from 2 to
$80 \mathrm{mM}(2,4,6,8,15,30,40,60,80 \mathrm{mM})$. The dispersions were maintained at a temperature just below boiling and stirred for $2 \mathrm{~min}$. For gelling temperature determination, the sample was used immediately for the NIR analysis. To prepare samples for other studies, the hot gellan dispersion was poured into preheated $\left(98^{\circ} \mathrm{C}\right)$ metal molds (i.d. $35 \mathrm{~mm}$, length $33 \mathrm{~mm}$ ) and maintain at room temperature (ca. $22^{\circ} \mathrm{C}$ ) or refrigerated temperature $\left(4 \pm 2{ }^{\circ} \mathrm{C}\right)$ until further analysis.

SW-NIR spectra were recorded in the diffuse reflectance mode with a fiber optic probe and a DPA-20 spectrophotometer (DSquared Development Inc., La Grande, OR) from 600 to $1100 \mathrm{~nm}$ at $0.5 \mathrm{~nm}$ intervals. The diameter of the fiber optic probe was about $1 \mathrm{~cm}$ (Huang et al., 2001). For spectral collection, the probe was placed in direct contact with the surface of the gel or immersed into the gellan dispersion. Except for gelling temperature determination, each individual spectrum was the mean of 20 scans with a $250 \mathrm{~ms}$ acquisition time for each scan. The spectra were collected at room temperature.

\subsubsection{Classifying commercial low and high acyl gellan gels by $\mathrm{LA} / \mathrm{HA}$ ratio}

One percent LA, HA, and mixtures of $1 \%$ 25/75 LA/HA, 50/50 LA/HA and 75/25 LA/HA with selected calcium concentrations $(2,4,6,8,15,30,40,60,80 \mathrm{mM})$ were prepared. After each heated gellan dispersion was poured into metal tubes, gels were maintained at room temperature for $24 \mathrm{~h}$ prior to NIR analysis. Two gels were prepared from each solution. For each gel, three spectra were recorded from selected positions (bottom center, bottom side and surface center of the cylinder gel). The selection of three different positions for NIR measurement is based upon the possibility that NIR spectral features may vary among these three positions and the variance among different positions in the same sample should be accounted for during the NIR model development. The cooling rate of gellan dispersion at the surface of the cylinder mold is normally faster than that at the bottom, which may cause differences in physical or chemical properties between gel formed at the surface and the bottom. In addition, spectra taken from the center of the cylinder gel may be slightly different to the one from the side of the cylinder gel because of peripheral effects.

\subsubsection{Storage time, temperature and properties of low acyl gellan gels}

A $1 \%$ LA gellan with $4 \mathrm{mM}$ calcium added was selected for this study. Gellan dispersions were prepared and poured into metal molds. The gels were set at room temperature (ca. $\left.22{ }^{\circ} \mathrm{C}\right)$ or stored in the refrigerator $\left(4 \pm 2{ }^{\circ} \mathrm{C}\right)$. NIR spectra were acquired after 5, 12, 24, 48 and $72 \mathrm{~h}$ for gels at room or refrigerated temperatures. Three gels were tested for each storage time and four spectra (two from the bottom center and two from the surface center of the cylinder gel) were acquired for each gel. Gels were immediately discarded after each test to avoid potential microbial contaminations that may affect gel properties. Each experiment was replicated. 


\subsubsection{Gelling temperature determination}

A $1 \%$ hot gellan dispersion (ca. $80^{\circ} \mathrm{C}$ ) with selected calcium concentrations $(4,14$ or $30 \mathrm{mM})$ was transferred into a $250 \mathrm{ml}$ beaker. Prior to spectral collection, a reference spectrum of Spectralon ${ }^{\circledR}$ (Labsphere, Inc., North Sutton, NH) was obtained. The Spectralon ${ }^{\circledR}$ was placed at the bottom of a beaker reflecting the SW-NIR light to the detector during spectral acquisition (Fig. 1). The reference spectrum was automatically subtracted from each gel spectrum by the instrument. Spectra were recorded by immersing the fiber optic probe into the gellan dispersion. The distance between the end of the probe and the surface of the Spectralon ${ }^{\circledR}$ was maintained constant at $20 \mathrm{~mm}$. Each spectrum was the mean of 10 scans with a $100 \mathrm{~ms}$ exposure time for each scan. The hot gellan dispersion was held at ambient temperature until it reached room temperature. Gel spectra were continuously recorded at about $0.5^{\circ} \mathrm{C}$ intervals as the temperature dropped. The temperature of each solution was determined with two thermocouple thermometers (type 421305, Extech Instruments Corp., Waltham, MA) and monitored continuously during spectral acquisition. One of the thermocouple probes was positioned at the surface of the Spectralon ${ }^{\circledR}$ and the other at the end of the fiber optic probe. A series of spectra (labeled continuously as spectrum index) with each spectra corresponding to two temperatures were recorded for each gellan sample as its temperature decreased from hot solution state to gelling state. The gelling temperature was designated as the temperature at which the sol-gel transition occurred with the spectrum taken at this transition point marked. Replicate experiments were conducted for each sample with selected calcium concentration.

\subsection{Data analysis}

Data analyses were conducted by using DeLight 2.2 software package (DSquared Development Inc., La Grande, OR). Prior to multivariate data analysis, spectra

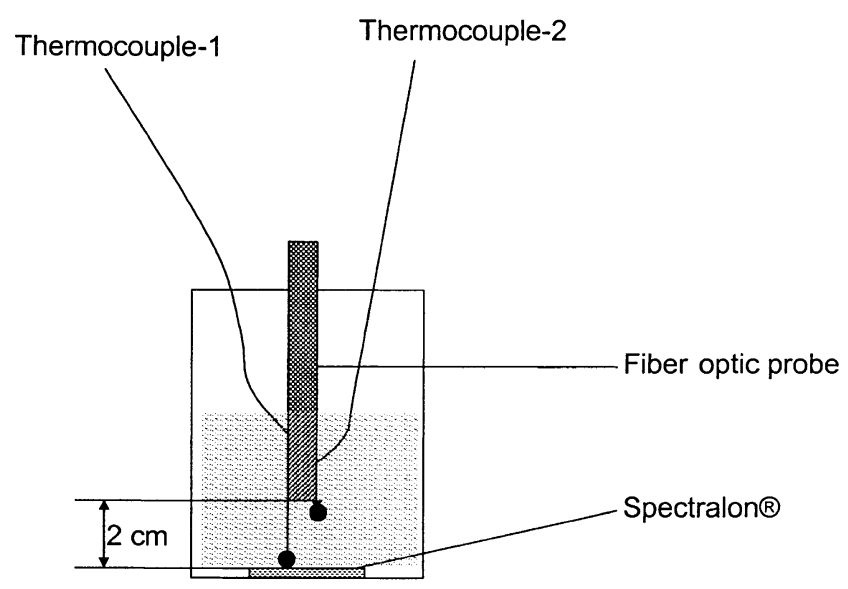

Fig. 1. Position of fiber optic probe, Spectralon ${ }^{\circledR}$ and thermocouples during NIR spectra acquisition for gelling temperature determination. pre-transformation such as bin, smooth and second derivative transformation or first order polynomial subtract were conducted to remove baseline shift and to increase the signal to noise ratio. Principal component analysis (PCA), evolving factor analysis (EFA) and partial least square regression (PLS) were selected to interpret NIR spectra.

PCA is a commonly used data compression technique that reduces data sets to selected few dimensions (principal components) that account for most of the variance within the data. EFA conducts a PCA analysis on a moving block of data and is usually very useful for analyzing time series data. PLS is a commonly used linear regression method for highly collinear data. PLS involves two steps of regression. The first step is to calculate a set of eigenvectors (latent variables) that account for the variance in the date set. The second step is to regress these vectors (latent variables) against the concentration of the analytes of interest (Geladi \& Kowalski, 1986; Martens \& Naes, 1987).

Leave-one-out-cross validation was used to verify the predictability of PLS models. In leave-one-out-cross validation, all samples except one are used to construct a model and then the model is used to predict the remained gel. After that, a second sample is left out from all samples and a newly constructed model is used to predict the sample. This procedure is repeated until each sample is left out and predicted by a model once. The $R^{2}$ of predicted values versus reference values, and the standard error of prediction were used to indicate the quality of the model. Except for the gelling temperature determination, spectra after the first polynomial subtraction were used for the PCA and second derivative transformations of spectra were used for PLS

\subsubsection{Classifying commercial low and high acyl gellan gels by $\mathrm{LA} / \mathrm{HA}$ ratio}

PCA was used to classify the five selected groups of LA, HA and mixtures of LA/HA gellan gel. PLS was selected to determine the percentage of HA in these five groups. Two latent variables were used for model construction.

\subsubsection{Storage time, temperature and the properties of low acyl gellan gels \\ PCA was used to classify gels at two selected temperatures and gels at selected storage times. PLS regression was also used to correlate the NIR spectra with gel storage time.}

\subsubsection{Gelling temperature determination}

PCA was performed to identify the principal components related to the gelling temperature and estimate the range of gelling temperature. The second derivative transformations of spectra were used for PCA analysis. After the range of gelling temperature was estimated, EFA was used to further identify specific spectra corresponding to the gelling temperature. Spectra after the first polynomial subtracting were used for the EFA. 


\section{Results and discussion}

\subsection{Classifying commercial low and high acyl gellan gels by $L A / H A$ ratio}

Figs. 2-4 present NIR spectra at room temperature after subtracting a first order polynomial to remove the baseline shifts. The absorption intensity of the $970 \mathrm{~nm}$ water band was significantly different between the LA and HA gels, with the LA gel exhibiting a much higher amplitude and sharper peak than the HA gel at the equivalent calcium concentrations (Fig. 2). Depending upon the ratio of LA to HA, the peak amplitude at $970 \mathrm{~nm}$ increased as the LA gel percentage increased. These differences in NIR spectral features reflect differences in water-hydrocolloid interactions in the structure of both LA and HA gels (Chandrasekaran \& Thailambal, 1990).

Fig. 3 presents spectra around the $970 \mathrm{~nm}$ water band for five selected groups of $1 \%$ gellan gel mixtures with nine selected calcium concentrations for each group. The observed spectra of the nine LA gels are differentiated based upon calcium concentration (Fig. 3). However, the nine spectra of HA gels overlapped and were difficult to differentiate. The results from Fig. 3 imply that changes in calcium concentration exhibited greater effects on the absorption intensity of LA gels than on the absorption intensity of HA gels in the primary water band region. These are consistent with the fact that LA gels are more sensitive to changes in calcium concentration than HA gels because the two gels follow different cation-driven mechanisms of gelation (Chandrasekaran \& Radha, 1995). In addition, changes in calcium concentration exhibited similar effects on the absorption intensity of the $970 \mathrm{~nm}$ water band in LA/ HA gel mixtures to that in HA gellan gels.

In LA gellan gels, except for the $80 \mathrm{mM}$ gel, the absorption intensity of the $970 \mathrm{~nm}$ water band region was inversely proportional to the calcium concentration (Fig. 4).

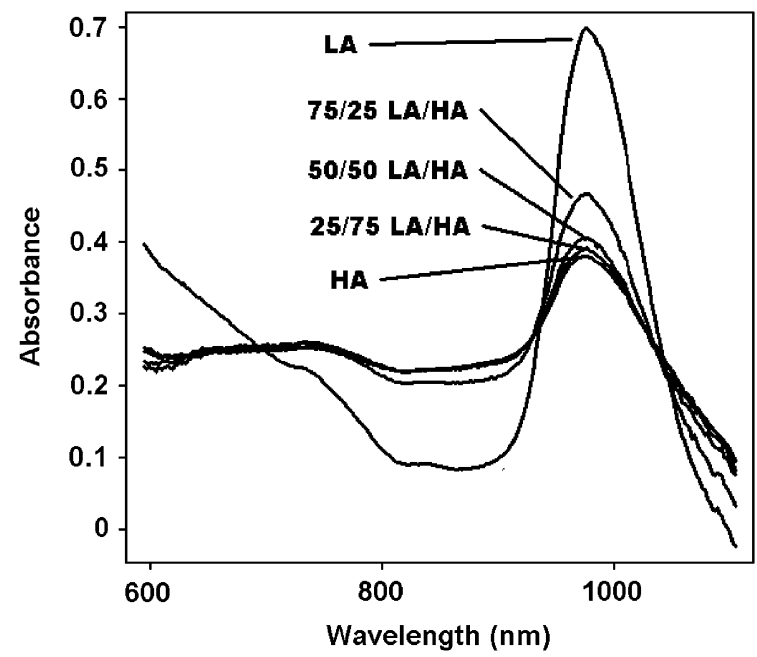

Fig. 2. Representative NIR spectra of $1 \%$ LA, HA and LA/HA gellan gel mixtures $\left(6 \mathrm{mM} \mathrm{Ca}^{++}\right)$at room temperature.

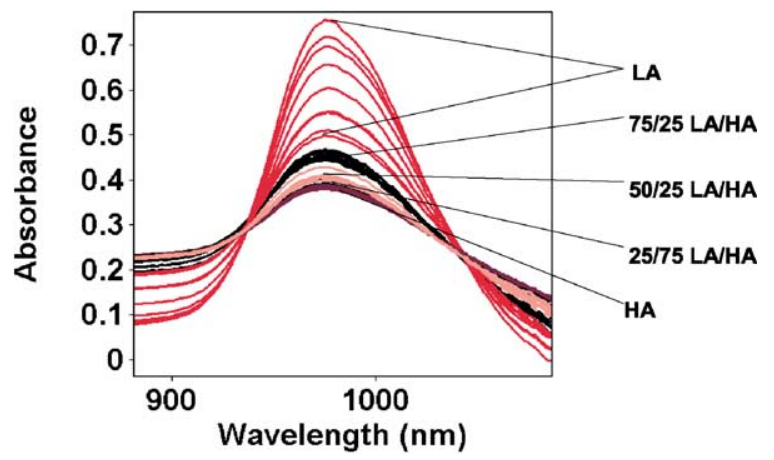

Fig. 3. Representative NIR spectra of five selected $1 \%$ LA/HA gellan gel mixtures (0-100\% HA) at nine selected calcium concentrations (2$80 \mathrm{mM})$ at room temperature.

No similar tendency was observed for HA gellan gels. The exception of the $80 \mathrm{mM}$ gel may reflect the special textural properties of a LA gellan gel with extremely high calcium concentrations in the gel (Mao, Tang, \& Swanson, 2001).

Gels were classified based upon relative concentration of HA and LA using PCA (Fig. 5). It is possible to classify five selected groups of gellan gels with nine selected calcium concentrations for each group. The four latent variables of PCA explained $96.98 \%$ of the variation in spectral features, with the first latent variable accounting for $80.27 \%$, the second $11.56 \%$, the third $4.01 \%$ and the fourth $1.14 \%$. Using only two latent variables, PCA was able to clearly classify HA and LA gels with 2-80 mM added calcium (Fig. 5). This means that the chemical or physical properties of LA and HA gels are quite different and that SW-NIR spectroscopy can detect such differences in the presence of different calcium concentrations in each group of gels.

HA gellan gels with selected calcium concentrations (2-80 $\mathrm{mM})$ exhibited a very tight data cluster in Fig. 5, while LA gellan gels $(2-80 \mathrm{mM})$ distributed in a much wider area. The distribution areas of three mixed gels with

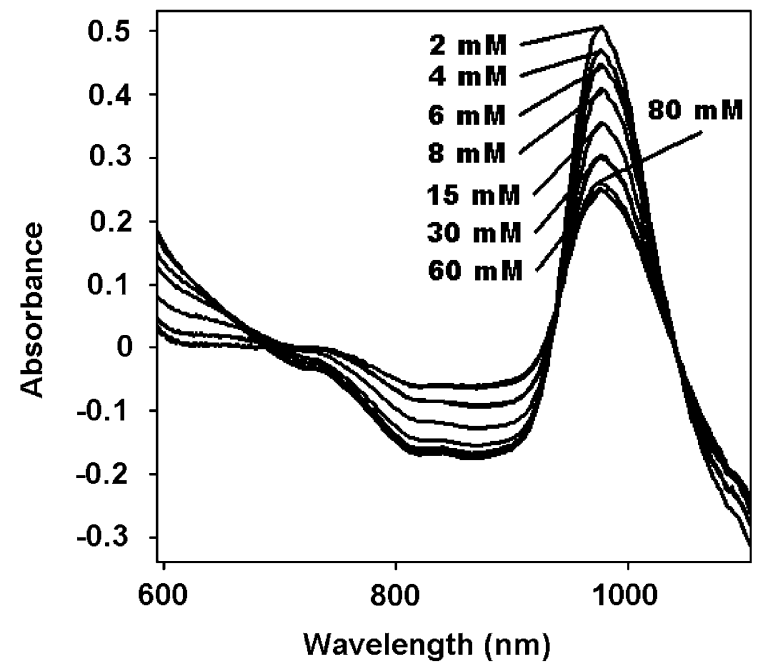

Fig. 4. Representative NIR spectra of $1 \%$ LA gellan gels with selected calcium concentrations $(2-80 \mathrm{mM})$ at room temperature. 


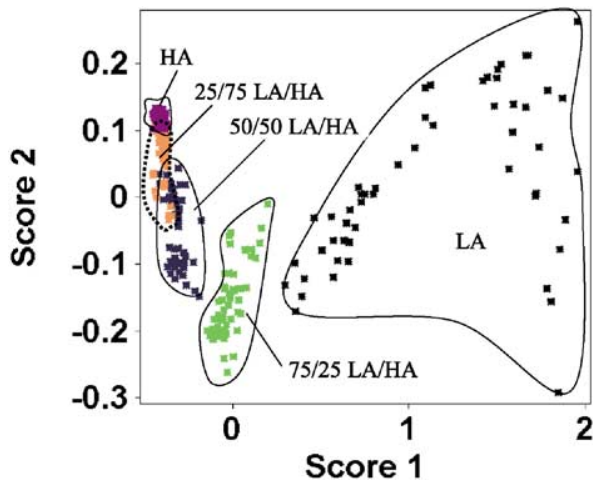

Fig. 5. PCA score plot of five selected 1\% LA/HA gellan gel mixtures $(0-100 \% \mathrm{HA})$ at nine selected calcium concentrations $(2-80 \mathrm{mM})$.

selected calcium concentration $(2-80 \mathrm{mM})$ were between that of LA and HA gellan gels. The difference in distribution area among five selected groups of gellan gels may also indicate that these five groups of gels have different sensitivity to changes in calcium concentrations.

There were some overlaps (Fig. 5) between HA, 25/75 LA/HA mixtures and 50/50 LA/HA mixtures. The samples overlapped between 50/50 LA/HA and 25/75 LA/HA mixtures were identified as gels with low calcium concentrations $(\leq 8 \mathrm{mM})$ for $50 / 50 \mathrm{LA} / \mathrm{HA}$ mixed gels and with high calcium concentrations ( $\geq 60 \mathrm{mM}$ ) for $25 / 75$ LA/HA mixed gels. Some gels of 25/75 LA/HA mixtures with low calcium concentration $(\leq 8 \mathrm{mM})$ overlapped with HA gels. The overlapping between HA, 25/75 LA/HA and $50 / 50 \mathrm{LA} / \mathrm{HA}$ mixtures indicate that the spectral features of these gels were very close and unable to differentiate with the PCA method.

PLS regression with two latent variables was used to predict the percentage of HA gels in five selected groups of gels with nine different calcium concentrations. The $R^{2}$ of the predicted values versus the actual values of percentage of the HA gellan gels was 0.93 (Fig. 6) and the standard error of prediction was $9.19 \%$.

The spectral differences between the two commercial LA and HA gels observed in this study were not necessarily a result of compositional differences in acetyl or glyceryl

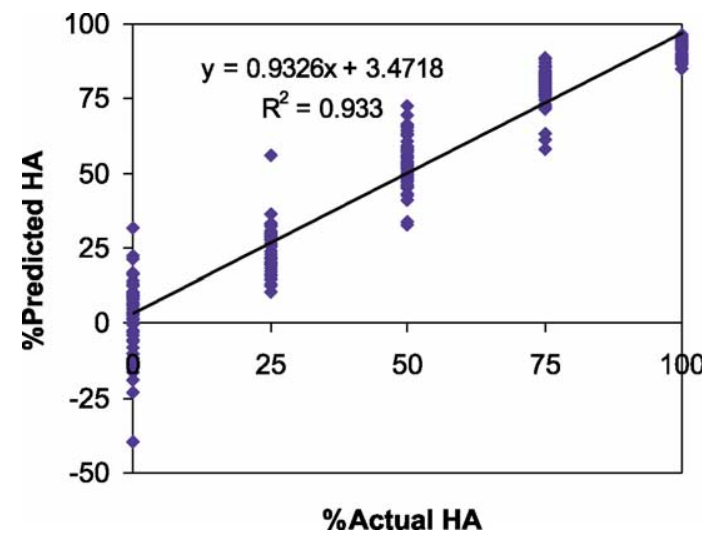

Fig. 6. Actual versus predicted values of HA gellan gel concentration in $1 \%$ LA/HA gellan gel mixtures $(0-100 \% \mathrm{HA})$.

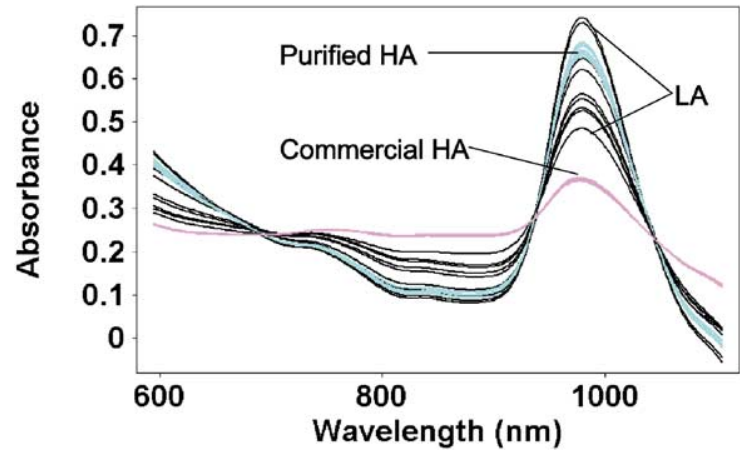

Fig. 7. Representative NIR spectra of $1 \%$ LA, commercial and purified gellan gel mixtures at selected calcium concentrations $(2-80 \mathrm{mM})$ at room temperature.

groups in these gels. The only commercial available HA gellan powder (LT 100) is not purified and contain residues such as protein, while the LA powder used in this study is purified. The residues such as protein in HA (LT 100) may account for some spectral differences between HA and LA gels observed in this study.

NIR spectral analysis of purified HA (EX-8593), provided by $\mathrm{CP}$ Kelco, indicated that the existence of residues in commercial HA significantly decreases the NIR spectral absorption intensity (Fig. 7). However, similar to the commercial HA gels, changes in calcium concentrations exhibited much less effect on the absorption intensity of purified HA gels than on that of LA gels in the primary water band region, indicating that both commercial and purified HA gels was less sensitive to changes in calcium concentration than LA gels.

\subsection{Storage time, temperature and the properties of low acyl gellan gels}

As previously reported for the infrared spectra of a pure water system, a change in temperature caused the position of the water bands to shift and spectral absorbance intensity to change (Finch \& Lippincott, 1956; Huang, 2001; Lin \& Brown, 1992; Wülfert, Kok, Noord, \& Smilde, 2000). The water band around $970 \mathrm{~nm}$ became sharper and shifted to

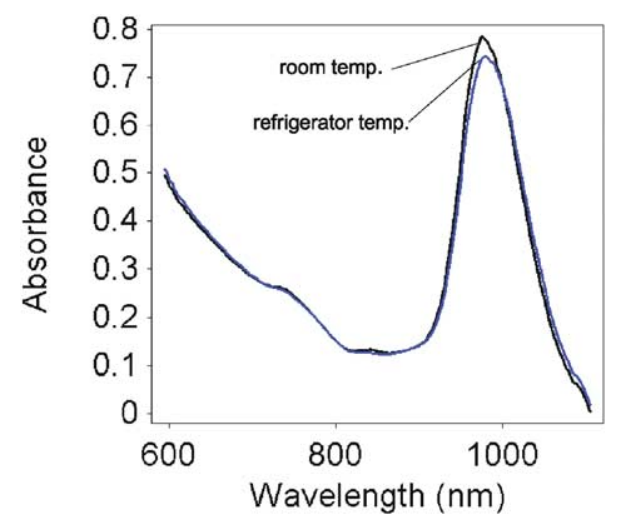

Fig. 8. NIR spectra of $1 \%$ LA gellan gel $\left(4 \mathrm{mM} \mathrm{Ca}^{++}\right)$at room temperature $\left(22{ }^{\circ} \mathrm{C}\right)$ and refrigerator temperature $\left(4{ }^{\circ} \mathrm{C}\right)$. 




Fig. 9. PCA results for $1 \% \mathrm{LA}$ gel $(4 \mathrm{mM}$ calcium) at room temperature $\left(22{ }^{\circ} \mathrm{C}\right)$ and refrigerator temperature $\left(4{ }^{\circ} \mathrm{C}\right)$.

a lower wavelength as temperature increased from the cold temperature $\left(4{ }^{\circ} \mathrm{C}\right)$ to room temperature $\left(22^{\circ} \mathrm{C}\right)$ (Fig. 8). Fig. 9 shows that $1 \%$ LA gellan gels with a $4 \mathrm{mM}$ calcium concentration stored at two different temperatures could be differentiated using PCA analysis with only one latent variable.

Neither PCA nor PLS could differentiate among samples stored at different time intervals $(5-72 \mathrm{~h})$, which suggested a minor or undetectable differences of NIR spectral features among these samples. This implies that storage time (5$72 \mathrm{~h}$ ) exhibits a limited effect on the water binding properties and gel structures of 1\% LA gellan gels with $4 \mathrm{mM}$ added calcium. The result is consistent with an earlier report (CP Kelco, 2000) that gellan gel reached a mature gel strength quickly once gelling temperature was reached and the gel structure remained stable under normal storage conditions.

\subsection{Gelling temperature determination}

During SW-NIR spectral acquisition, temperature variations were observed at different positions within the gel or gellan dispersion because the heat transfer rate was not uniform in the gel or dispersion. For gellan dispersion at approximately $70{ }^{\circ} \mathrm{C}$, the temperature gradient between the surface of the Spectralon ${ }^{\circledR}$ in contact with the gel and the end of the fiber optic probe (a $2 \mathrm{~cm}$ distance away) could be as large as $7{ }^{\circ} \mathrm{C}$, while for gellan solution at around $40{ }^{\circ} \mathrm{C}$, the difference could be $3{ }^{\circ} \mathrm{C}$. The magnitude of this temperature gradient depended upon the sample temperature, the state of sample (solution or gelling state), and the calcium concentration in the sample.

Despite the difficulties in achieving uniform temperature within the experimental system, the gelling temperature of LA gellan gels could be estimated using a PCA with only two principal components. For $1 \%$ gellan at three different calcium concentrations (4, 14 and $30 \mathrm{mM}$ ), PCA results were consistent (Fig. 10). For each gel, the PCA score corresponding to the first principal component (Score 1) increased with increasing sample spectrum index, which corresponds to a decrease in sample temperature. Therefore, the first principal component may directly reflect a sample temperature change. The PCA score corresponding to the second principal component (Score 2) decreased initially and then increased as spectrum index increased or temperature decreased, indicating that the second principal component was not related to temperature but to another factor. When a hot gel solution is cooled to room temperature, two obvious changes occur in the sample. The first one is a temperature decrease and the second, a sol-gel phase transition. In addition, increasing the number of principal components for the PCA model did not help to account for the spectral variance of a gel with decreasing temperatures, which means that there were no other principal components except the second one accounting for the phase transition of the gel. The minimum observed in the plot of Score 2 versus gel spectrum index would correspond with the phase transition of the sample (Fig. 10(b)).

From Fig. 10(b), the estimated gelling point corresponded with spectrum index-45. The exact gelling temperature was pinpointed by combining the results of PCA with EFA using one latent variable. From the plots of score of EFA, spectrum index-47 (corresponding temperature was 40.1 and $43.1^{\circ} \mathrm{C}$ ) was identified as the spectrum corresponding to the sol-gel transition (Fig. 11).

Table 1 shows gelling temperatures for 1\% LA gellan with three selected calcium concentrations. The gelling temperatures from our study, especially for the temperatures
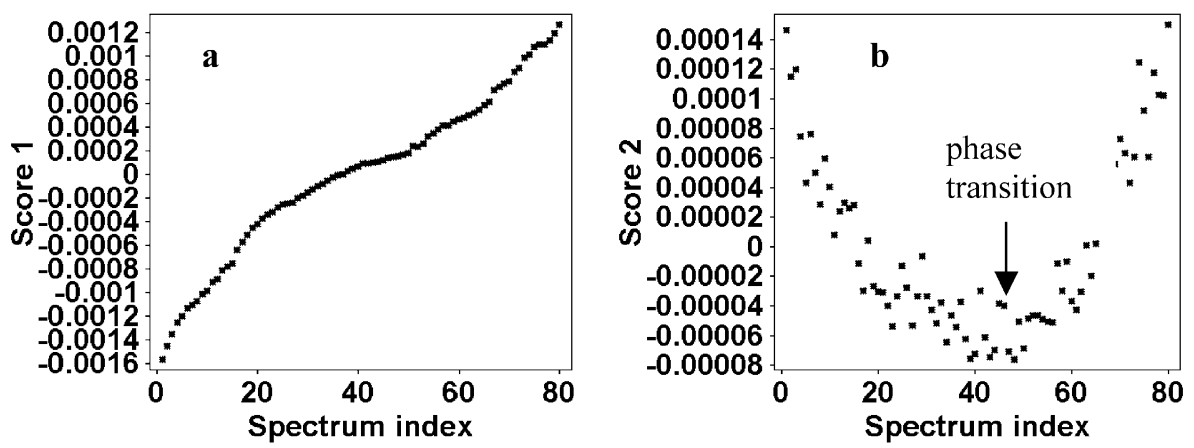

Fig. 10. Score 1 (a) and Score 2 (b) from PCA versus spectrum indices for 4 mM LA gellan gel. 


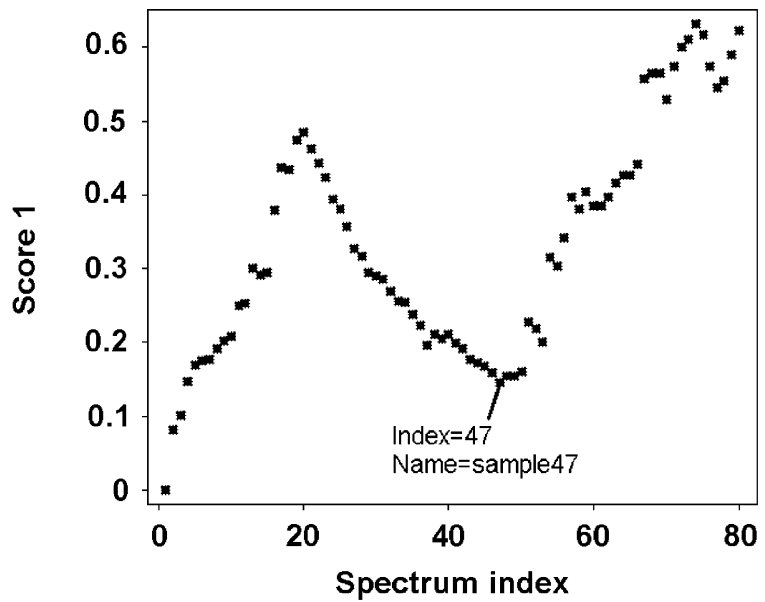

Fig. 11. Score 1 from EFA versus spectrum indices for $4 \mathrm{mM}$ LA gellan gel.

Table 1

Gelling temperature of $1 \%$ LA gellan by SW-NIR and other methods

\begin{tabular}{llllll}
\hline & NIR & & & \multicolumn{2}{l}{ Other $^{\mathrm{a}}$} \\
& Sample 1 & Sample 2 & & Dynamic & Visual \\
\hline $4 \mathrm{mM}$ & $40.1^{\mathrm{b}}(43.1)^{\mathrm{c}}$ & $40.0(42.2)$ & & 40.0 & 39.5 \\
$14 \mathrm{mM}$ & $52.0(55.6)$ & $52.3(55.5)$ & & 53.5 & 52.0 \\
$30 \mathrm{mM}$ & $62.3(66.1)$ & $62.8(63.5)$ & & 64.0 & 62.0 \\
\hline
\end{tabular}

${ }^{a}$ Data from Tang et al. (1997). Results of dynamic testing method and direct visual observation were provided.

b Temperature indicated by the thermocouple close to the Spectralon ${ }^{\circledR}$.

c Temperature indicated by the thermocouple close to the fiber optic probe.

indicated by the thermocouple close to the Spectralon ${ }^{\circledR}$, were similar to the gelling temperatures determined with dynamic rheological testing and direct visual observation reported by Tang, Tung, and Zeng (1997). During NIR spectral acquisition, the sample temperature close to the Spectralon ${ }^{\circledR}$ was always lower than the temperature close to the fiber optic probe, which means the gel solution close to the Spectralon ${ }^{\circledR}$ gelled faster than the portion close to the fiber optic probe. From Table 1, the gelling temperature detected by the thermocouple close to the Spectralon ${ }^{\circledR}$ was closer to the results of Tang, Tung, and Zeng (1997), which indicates that the NIR method is sensitive to detect the initiation of the sol-gel transition of LA gels.

\section{Conclusion}

SW-NIR demonstrates great promise for rapid determination of physical properties and chemical composition of gellan gels both in terms of the percentage of HA in a LA/HA mixture $\left(R^{2}=0.93\right.$, standard error $\left.=9.19 \%\right)$ and gelling temperature. Both gel composition and gelling temperature are often difficult to predict using other methods. SW-NIR can classify gels stored at different temperatures. SW-NIR may predict the time required for a gel to reach a mature strength following polymerization and stability of the gel during storage.

\section{Acknowledgements}

This research was supported by the USDA NRI Competitive Grant Program (NRI-2001-3550310770 and NRI-2001-35503-10033). We thank CP Kelco, Inc. for providing gellan polymer. Special thanks go to Dr Davis M. Mayes, DSquared Development, Inc. for assistance with the NIR analysis and data analysis.

\section{References}

Camacho, W., \& Karlsson, S. (2001). NIR, DSC, and FTIR as quantitative methods for compositional analysis of blends of polymers obtained from recycled mixed plastic waste. Polymer Engineering and Science, 41(9), 1626-1635.

Chandrasekaran, R., \& Radha, A. (1995). Molecular architectures and functional properties of gellan gum and related polysaccharides. Trends in Food Science and Technology, 6, 143-148.

Chandrasekaran, R., \& Thailambal, V. G. (1990). The influence of calcium ions, acetate and L-glycerate groups on the gellan double-helix. Carbohydrate Polymers, 12, 431-442.

CP Kelco (2000). Kelcogel gellan gum-an effective and useful gelling agent for foods (bulletin)

Finch, J. N., \& Lippincott, E. R. (1956). Hydrogen bond systemtemperature dependence of $\mathrm{OH}$ frequency shifts and $\mathrm{ON}$ band intensities. The Journal of Chemical Physics, 24, 908-909.

Geladi, P., \& Kowalski, B. R. (1986). Partial least-square regression: A tutorial. Analytica Chimica Acta, 185, 1-17.

Huang, Y. (2001). Non-destructive analysis of salt and moisture in food products by short-wavelength near-infrared (SW-NIR) spectroscopy [dissertation]. Pullman, WA: Washington State University.

Huang, Y., Cavinato, A. G., Mayes, D. M., Bledsoe, G. E., \& Rasco, B. A (2002). Non-destructive prediction of moisture and sodium chloride in cold smoked Atlantic salmon (salo salar). Journal of Food Science, 67(7), 2543-2547.

Huang, Y., Rogers, T. M., Wenz, M. A., Cavinato, A. G., Mayes, D. M., Bledsoe, G. E., \& Rasco, B. A. (2001). Detection of sodium chloride and moisture in cured salmon roe by SW-NIR spectroscopy. Journal of Agricultural and Food Chemistry, 49, 4161-4167.

Lin, J., \& Brown, C. W. (1992). Near-IR spectroscopic determination of $\mathrm{NaCl}$ in aqueous solutions. Journal of Applied Spectroscopy, 46, 1809-1815.

Mao, R., Tang, J., \& Swanson, B. G. (2001). Water holding capacity and microstructure of gellan gels. Carbohydrate Polymers, 46, $365-371$.

Martens, H., \& Naes, T. (1987). Multivariate calibration by data compression. In P. Williams, \& K. Norris (Eds.), Near-infrared technology in the agricultural and food industries (pp. 57-82). St Paul, MN: American Association of Cereal Chemists, Inc.

Nees, F. W., \& Buback, M. (1976). Study of the high pressure polymerization if ethylene by near infrared spectroscopy. The Journal of Physical Chemistry, 80(10), 1017-1023.

Phelan, M. K., Barlow, C. H., Kelly, J. J., Jinguji, T. M., \& Callis, J. B. (1989). Measurement of caustic and caustic brine solutions by spectroscopic detection of the hydroxide ion in 
the near-infrared region, 700-1150 nm. Analytical Chemistry, 61 1419-1424.

Rohe, T., Kolle, S., Stern, C., Eisenreich, N., \& Eyerer, P. (2001). Inline near infrared (NIR) spectroscopy for application in polymer extrusion processes. Recent Research in Developments in Pure and Applied Analytical Chemistry, 3(Pt. 1), 13-26.

Soper, S. A., \& Legendre, B. L. (1998). Single-molecule detection in the near-IR using continuous-wave diode laser excitation with an avalanche photon detector. Journal of Applied Spectroscopy, 52(1), 1-6.
Tang, J., Tung, M. A., \& Zeng, Y. (1997). Gelling temperature of gellan solutions containing calcium ions. Journal of Food Science, 62(2), 276-280.

Vieira, R. A. M., Sayer, C., Lima, E. L., \& Pinto, J. C. (2001). Detection of monomer droplets in a polymer latex by near-infrared spectroscopy. Polymer, 42(21), 8901-8906.

Wülfert, F., Kok, W. T., Noord, O. E., \& Smilde, A. K. (2000). Correction of temperature-induced spectral variation by continuous piecewise direct standardization. Analytical Chemistry, 72(7), 1639-1644. 